\title{
ESPACES TWISTORIELS ET STRUCTURES COMPLEXES NON STANDARDS
}

\author{
Guillaume Deschamps
}

\begin{abstract}
In this article we use the twistor theory in order to build "non standard" complex structures (with a meaning which we define) on the products of 4-manifolds with the sphere of dimension two. To that end, we enumerate the set of complex surfaces whose twistor space is $\mathcal{C}^{\infty}$-trivial. Among these surface we will study those which admit an anti-self-dual riemannian metric.
\end{abstract}

\section{Introduction}

En regardant le quotient du fibré $\mathcal{O}(1) \oplus \mathcal{O}(1) \longrightarrow \mathbb{C} P^{1}$ par une action holomorphe de $\mathbb{Z}^{4}$ bien choisie, Blanchard $[\mathbf{5}]$ a mis une structure complexe non kählerienne sur le produit $\mathbb{T}^{4} \times \mathbb{S}^{2}$ d'un tore $\mathbb{T}^{4}$ avec la sphère $\mathbb{S}^{2}$. Cette structure complexe n'est pas une déformation d'une structure complexe produit. Cet exemple est un cas particulier d'espace twistoriel : si $(M, g)$ est une 4-variété riemannienne orientée, on peut lui associer son espace twistoriel $\tau(M, g)$ qui est le fibré des structures presque complexes sur $T M$ compatibles avec l'orientation et la métrique [1]. C'est un fibré lisse localement trivial de fibre la sphère $\mathbb{S}^{2}$ et de groupe structural $S O(3)$. L'espace twistoriel $\tau(M, g)$ est muni d'une structure presque complexe canonique qui ne dépend que de la classe conforme de $g$. Cette dernière est intégrable si et seulement si la métrique $g$ est anti-autoduale [1]. On rappelle qu'une métrique $g$ sur une 4-variété est dite anti-autoduale si le tenseur de Weyl associé $W$, considéré comme un endomorphisme de l'ensemble des 2-formes, vérifie $\star W=-W$ où $\star$ est l'opérateur de Hodge. Dans l'exemple de Blanchard la structure complexe non kählerienne sur $\mathbb{T}^{4} \times \mathbb{S}^{2}$ est précisément la structure d'espace twistoriel d'un tore plat : $\tau\left(\mathbb{T}^{4}, g_{\text {plat }}\right) \simeq \mathbb{T}^{4} \times \mathbb{S}^{2}$.

2000 Mathematics Subject Classification. 53C28, 51M99, 53C27.

Key words. Twistor space, self-dual metric, spin structure, Enriques-Kodaira classification, almost hypercomplex manifold, parallelisable complex surface. 
On sait qu'une surface $K 3$ munie de sa métrique de Calabi-Yau ou que la surface $\mathbb{S}^{1} \times \mathbb{S}^{3}$ munie de sa métrique produit sont des exemples qui généralisent ceux de Blanchard. C'est-à-dire que ce sont des surfaces complexes $M$ dont la structure d'espace twistoriel munit le produit $M \times \mathbb{S}^{2}$ d'une structure complexe qui n'est pas une déformation de la structure complexe produit. Le but de cet article est de donner une classification des variétés qui généralisent celles de Blanchard. C'est-à-dire de déterminer la liste des 4 -variétés riemanniennes orientées $(M, g)$ munit d'une structure complexe, dont la fibration twistorielle est $\mathcal{C}^{\infty}$-triviale et telle que la métrique $g$ est anti-autoduale. Pour de telles variétés, nous verrons que la structure complexe d'espace twistoriel n'est jamais une déformation de la structure complexe produit, ni biméromorphe à une variété kählerienne [11].

Nous commencerons par donner un critère simple pour savoir si la fibration twistorielle d'une 4 -variété est $\mathcal{C}^{\infty}$-triviale (Partie 2). Nous donnerons alors la liste des surfaces complexes à fibré twistoriel trivial. Pour cela il nous faudra d'abord déterminer quelles surfaces complexes possèdent une structure spin. On précisera ainsi un résultat de Yau [37] en donnant l'ensemble des 4-variétés parallélisables munies d'une structure complexe. Tous ces résultats sont regroupés dans le Théorème 1. Dans la Partie 3 nous donnerons une résolution quasi-complète à notre problème. Ce théorème peut être vu comme une version riemannienne d'un résultat de Boyer [8, Theorem 3] (lire aussi [17] et [29]).

Théorème 2. Soit $M$ une surface compacte complexe dont le fibré twistoriel est $\mathcal{C}^{\infty}$-trivial. Si la variété $M$ peut être munie d'une métrique riemannienne anti-autoduale alors $M$ est:

a) une surface $K 3$,

b) un tore,

c) une surface hyperelliptique,

d) une surface de Hopf,

e) une surface minimale spin de dimension de Kodaira un.

Réciproquement dans les cas a), b), c) et d) il existe toujours une métrique riemannienne anti-autoduale.

Comme application, nous donnerons une famille d'exemples de 4-variétés $M$ sans structure complexe dont le produit $M \times \mathbb{C} P^{1}$ est complexe. C'est en quelque sorte une structure complexe "non standard" (Partie 4).

Théorème 3. Pour toute 3-variété compacte hyperbolique orientable $H$, il existe une structure complexe sur le produit $H \times \mathbb{S}^{1} \times \mathbb{S}^{2}$ bien que $H \times \mathbb{S}^{1}$ n'en possède pas. 
Enfin pour certaines surfaces $M$ du Théorème 2 nous construirons une infinité de structures complexes non kähleriennes sur le produit $M \times \mathbb{S}^{2}$ qui ne sont pas déformations les unes des autres (Partie 5).

\section{Fibré twistoriel trivial}

Soit $(M, g)$ une 4-variété riemannienne orientée. On note $\bigwedge^{+}$le fibré des 2-formes autoduales, c'est-à-dire invariantes par l'opérateur de Hodge $\star$. La métrique sur $M$ induit une métrique sur le fibré $\wedge^{+}$. L'espace twistoriel $\tau(M, g)$ de $(M, g)$ est par définition le fibré unitaire $\mathbb{S}\left(\bigwedge^{+}\right)$. À difféomorphisme près la fibration twistorielle ne dépend que de l'orientation de $M[\mathbf{1}],[\mathbf{1 2}]$.

Définition. Une 4-variété orientée $M$ est presque hypercomplexe s'il existe trois structures presque complexes $I, J$ et $K$ qui respectent l'orientation et telles que $I J=-J I=K$.

En d'autres termes $M$ est presque hypercomplexe si le groupe structural du fibré tangent de $M$ se réduit à $S U(2)$.

Proposition 1. Le fibré twistoriel d'une 4-variété orientée est trivial si et seulement si $M$ est presque hypercomplexe.

Preuve: Le groupe des difféomorphismes de la sphère $\mathbb{S}^{2}$ qui préservent l'orientation Diff ${ }^{+} \mathbb{S}^{2}$ se rétracte fortement sur le groupe spécial orthogonal $S O(3)[33]$. Par conséquent le fibré twistoriel $\mathbb{S}\left(\bigwedge^{+}\right) \longrightarrow M$ est trivial en tant que Diff ${ }^{+} \mathbb{S}^{2}$-fibré si et seulement s'il est trivial en tant que $S O(3)$-fibré et donc si et seulement si le fibré des 2-formes autoduales $\bigwedge^{+} \longrightarrow M$ l'est. Dans ce cas on peut définir une base orthonormée de champs de vecteurs sur $\Lambda^{+}$, ce qui revient précisément à se donner une structure presque hypercomplexe sur $(M, g)$.

Proposition 2. Une 4-variété $M$ est parallélisable si et seulement si elle est presque hypercomplexe et de caractéristique d'Euler nulle.

Preuve: Si $X$ est un champ de vecteurs partout non nul, $(X, I X, J X, K X)$ est une base de champs de vecteurs de $M$.

L'intérêt de la Proposition 1 vient du fait qu'on sait caractériser les 4-variétés presque hypercomplexes. On a ainsi une caractérisation topologique des 4-variétés à fibré twistoriel trivial.

Théorème $([\mathbf{3 6}])$. Une 4-variété orientée $M$ est presque hypercomplexe si et seulement si $M$ est spin et si sa caractéristique d'Euler $\chi$ et sa signature $\tau$ vérifient la relation : $3 \tau+2 \chi=0$. 
Pour déterminer la liste des surfaces complexes dont le fibré twistoriel est trivial il suffit de connaître la liste des surfaces complexes qui sont spin et de regarder celles dont la première classe de Chern vérifie $c_{1}^{2}=0$. En utilisant la Proposition 2 on aura également la liste des surfaces complexes qui sont parallélisables en tant que 4-variétés réelles.

Si $M$ est une 4-variété spin alors sa forme d'intersection est paire [2]. Une surface complexe spin est donc minimale c'est-à-dire sans courbe exceptionnelle [17].

On dit qu'une surface complexe $M$ est dans la classe $V I I_{0}$ si elle est minimale, si sa dimension de Kodaira est $-\infty$ et si son premier nombre de Betti est 1. Bogomolov [6] puis Teleman [34] ont montré que les seules surfaces de la classe $V I I_{0}$ dont le deuxième nombre de Betti $b_{2}$ est nul sont les surfaces de Hopf et les surfaces d'Inoue [19]. On connaît des exemples de surfaces dans la classe $V I I_{0}$ avec $b_{2}>0$. On parlera de la classe $V I I_{0}^{+}$.

Théorème 1. Le tableau suivant donne la liste des surfaces complexes qui sont spin, qui sont à fibré twistoriel trivial ainsi que celles qui sont parallélisables.

\begin{tabular}{|c|c|c|c|c|}
\hline $\begin{array}{c}\text { Dimension } \\
\text { de } \\
\text { Kodaira }\end{array}$ & Surfaces & $\begin{array}{c}\text { Surfaces } \\
\text { spin }\end{array}$ & $\begin{array}{c}\text { Surfaces } \\
\text { à fibré } \\
\text { twistoriel } \\
\text { trivial }\end{array}$ & $\begin{array}{c}\text { Surfaces } \\
\text { parallélisable } \\
\text { sur } \mathbb{R}\end{array}$ \\
\hline \multirow{5}{*}{$\begin{array}{c}\text { les surfaces de Hopf } \\
\text { les surfaces d'Inoue } \\
\text { les surfaces } \\
\text { de la classe } V I I_{0}^{+} \\
\text {le plan projectif } \\
\text { complexe } \mathbb{C} P^{2} \\
\text { les surfaces réglées } \\
\text { de genre } g \text { dont la } \\
\text { forme d'intersection } \\
\text { est paire }\end{array}$} & non & oui & oui & oui \\
& $\begin{array}{c}\text { non } \\
\text { les surfaces réglées } \\
\text { de genre } g \text { dont la } \\
\text { forme d'intersection } \\
\text { est impaire }\end{array}$ & non & non & non \\
& & non & si $g=1$ \\
\hline
\end{tabular}




\begin{tabular}{|c|c|c|c|c|}
\hline 0 & $\begin{array}{c}\text { les tores } \\
\text { les surfaces } \\
\text { hyperelliptiques } \\
\text { les surfaces de } \\
\text { Kodaira primaires } \\
\text { les surfaces de } \\
\text { Kodaira secondaires } \\
\text { les surfaces de } K 3 \\
\text { les surfaces d'Enriques }\end{array}$ & $\begin{array}{c}\text { oui } \\
\text { oui } \\
\text { oui } \\
\text { cf. } \\
\text { remarque } \\
\text { oui } \\
\text { non }\end{array}$ & $\begin{array}{c}\text { oui } \\
\text { oui } \\
\text { oui } \\
\text { celles qui } \\
\text { sont spin } \\
\text { oui } \\
\text { non }\end{array}$ & $\begin{array}{c}\text { oui } \\
\text { oui } \\
\text { oui } \\
\text { celles qui } \\
\text { sont spin } \\
\text { non } \\
\text { non }\end{array}$ \\
\hline 1 & $\begin{array}{c}\text { les surfaces minimales } \\
\text { avec premier nombre } \\
\text { de Betti impair } \\
\text { les surfaces minimales } \\
\text { avec premier nombre } \\
\text { de Betti pair }\end{array}$ & $\begin{array}{l}\text { certaines } \\
\text { certaines }\end{array}$ & $\begin{array}{l}\text { celles qui } \\
\text { sont spin } \\
\text { celles qui } \\
\text { sont spin }\end{array}$ & $\begin{array}{c}\text { celles qui } \\
\text { sont spin } \\
\text { celles qui sont spin } \\
\text { et de caractéristique } \\
\text { d'Euler nulle }\end{array}$ \\
\hline 2 & $\begin{array}{c}\text { les surfaces } \\
\text { de type général }\end{array}$ & cetaines & aucune & aucune \\
\hline
\end{tabular}

Remarque. Les surfaces de Kodaira secondaires sont des quotients finis des surfaces de Kodaira primaires par un groupe $G$ d'ordre 2, 3, 4 ou 6 dont l'action est propre, discontinue et sans point fixe [15], [2]. On n'a pas de classification de ces surfaces. Mais on peut construire de nombreux exemples qui sont spin. Ainsi lorsque $S$ est un quotient d'ordre 3 d'une surface de Kodaira primaire, son fibré canonique $K_{S}$ est tel que $K_{S}^{\otimes 3}$ est trivial. Sa première classe de Chern $c_{1}(S)$ vérifie donc $3 c_{1}(S)=0$ : la surface $S$ est spin. Pour les surfaces elliptiques de dimension de Kodaira 1, il existe quelques caractérisations de celles qui sont spin en fonction des multiplicités des fibres multiples [15]. La propriété d'admettre une structure spin n'étant pas invariante par passage au quotient il n'est pas raisonnable d'espérer donner la liste des surfaces proprement elliptiques ou de type général qui sont spin.

\subsection{Démonstration du Théorème 1 .}

Soit $b_{2}$ le deuxième nombre de Betti et $\tau$ la signature d'une surface complexe $M$. On note $b^{+}$(respectivement $b^{-}$) la dimension maximale du sous-espace sur lequel la forme d'intersection est positive (respectivement négative). Par définition on a d'une part $b_{2}=b^{+}+b^{-}$et d'autre part $\tau=b^{+}-b^{-}$. Si $M$ est une surface de la classe $V I I_{0}^{+}$on a $b_{2}>0$ et $b^{+}=0[\mathbf{1 5}],[\mathbf{2}]$. Le théorème de Donaldson [14] nous assure alors que 
la forme d'intersection de $M$ n'est pas paire, en particulier $M$ n'est pas spin.

On note $\Sigma_{g}$ la surface de Riemann de genre $g$. Les surfaces réglées de genre $g$ dont la forme d'intersection est paire sont toutes des déformations du produit $\Sigma_{g} \times \mathbb{C} P^{1}[\mathbf{1 5}],[\mathbf{2}]$ en particulier elles sont spin. De plus on a $c_{1}^{2}=8(1-g)$ et $\chi=4(1-g)$. Parmi celles-ci seules les surfaces réglées de genre 1 sont à fibré twistoriel trivial et parallélisables.

La signature d'une surface d'Enriques est -8, ce n'est pas un multiple de 16. Par le théorème de Rohlin [30] on en déduit que les surfaces d'Enriques ne sont pas spin.

Les surfaces de Kodaira primaires et les surfaces $K 3$ sont spin puisque par définition leur fibré canonique est trivial. Les surfaces de Hopf primaires sont par définition difféomorphes à $\mathbb{S}^{1} \times \mathbb{S}^{3}$. Elles sont spin puisque parallélisables.

Reste à montrer que les surfaces de Hopf secondaires, les surfaces d'Inoue et les surfaces hyperelliptiques sont spin.

\section{Surfaces de Hopf secondaires.}

1. Description des surfaces de Hopf secondaires : Une surface de Hopf secondaire $S$ est un quotient de $\mathbb{C}^{2 \star}=\mathbb{C}^{2}-\{(0,0)\}$ par un groupe d'automorphismes $G$ dont l'action est propre, discontinue et sans point fixe : $S=\mathbb{C}^{2 \star} / G$. On cherche à montrer que toutes ces surfaces admettent une structure spin. Pour cela on commence par rappeler les principaux résultats de Kato sur la classification des surfaces de Hopf secondaires [21], [23], [28].

On note $\left(z_{1}, z_{2}\right)$ un système de coordonnées sur $\mathbb{C}^{2}$. ̀̀ biholomorphisme près, on peut supposer [21] que $G \subset G L\left(\mathbb{C}^{2}\right)$ sauf si $G$ est le groupe engendré par les applications :

$$
\left\{\begin{array}{l}
g\left(z_{1}, z_{2}\right)=\left(\gamma^{n} z_{1}+\lambda z_{2}^{n}, \gamma z_{2}\right) \\
h\left(z_{1}, z_{2}\right)=\left(\alpha^{n} z_{1}, \alpha z_{2}\right)
\end{array}\right.
$$

où :

$$
\left\{\begin{array}{l}
(\lambda, \gamma, \alpha) \in \mathbb{C}^{3} \\
0<|\gamma|<1 \\
\alpha \text { est une racine primitive } m^{\text {ième }} \text { de l'unité } \\
m, n \text { sont des entiers premiers entre eux et } m, n \geq 2 .
\end{array}\right.
$$

Mais dans ce cas on peut déformer ces surfaces et se ramener à $\lambda=$ $0[21]$. À difféomorphisme près il suffit donc d'étudier les surfaces de 
Hopf secondaires pour lesquelles $G \subset G L\left(\mathbb{C}^{2}\right)$. On note $H=\{g \in$ $G /|\operatorname{det}(g)|=1\}$. On a alors la suite exacte :

$$
1 \longrightarrow H \longrightarrow G \longrightarrow \mathbb{Z} \longrightarrow 1 \text {. }
$$

Définition. On dit que $G$ est décomposable si $G=\mathbb{Z} \times H$. Sinon on dira que $G$ est indécomposable.

Si $G$ est décomposable, la surface de Hopf associée est difféomorphe au produit $\mathbb{S}^{1} \times \mathbb{S}^{3} / H[\mathbf{2 1}]$. Lorsque $G$ est indécomposable, la variété $S=\mathbb{C}^{2 \star} / G$ est un fibré au-dessus de $\mathbb{S}^{1}$ dont la fibre est difféomorphe à $\mathbb{S}^{3} / H$. On étudie ce cas en détail.

On utilise les notations suivantes pour les sous-groupes de $U(2)$ d'ordre fini :

- $B_{2^{k}(2 l+1)}^{\prime} \subset U(2)$ est le groupe engendré par les matrices $\left(\begin{array}{ll}0 & \delta \\ \delta & 0\end{array}\right)$ et $\left(\begin{array}{cc}\epsilon & 0 \\ 0 & \epsilon^{-1}\end{array}\right)$ avec $\delta, \epsilon \in \mathbb{C}$ d'ordre $2^{k}$ et $2 l+1$.

- $B_{n} \subset S U(2)$ est le groupe diédral d'ordre $4 n$. Il est engendré par les matrices $\left(\begin{array}{ll}0 & i \\ i & 0\end{array}\right)$ et $\left(\begin{array}{cc}e^{\frac{i \pi}{n}} & 0 \\ 0 & e^{\frac{-i \pi}{n}}\end{array}\right)$.

- $C \subset S U(2)$ est le groupe tetraédral. C'est le groupe d'ordre 24 engendré par les matrices $\left(\begin{array}{cc}i & 0 \\ 0 & -i\end{array}\right)$ et $\frac{1}{\sqrt{2}}\left(\begin{array}{cc}e^{\frac{3 i \pi}{4}} & e^{\frac{3 i \pi}{4}} \\ e^{\frac{i 2 \pi}{4}} & -e^{\frac{i \pi}{4}}\end{array}\right)$.

On note $\gamma$ un nombre complexe dont le module appartient à l'intervalle ]0, 1 et $\alpha$ une racine primitive $m^{\text {ième }}$ de l'unité.

Si $G$ est indécomposable alors dans les cas (D1) jusqu'à (D5) (voir ci-dessous) le groupe $G$ est :

$$
G=G_{0} \cup(\gamma u) G_{0} \text { avec } G_{0}=\left\langle\gamma^{2} \mathrm{Id}\right\rangle \times H .
$$

Dans le cas (D6) le groupe $G$ est :

$$
G=G_{0} \cup(\gamma u) G_{0} \cup(\gamma u)^{2} G_{0} \text { avec } G_{0}=\left\langle\gamma^{3} \mathrm{Id}\right\rangle \times H .
$$

Voici les différents cas qui peuvent arriver :

(D1) $H$ est le sous-groupe de $U(2)$ engendré par la matrice $\left(\begin{array}{cc}\alpha & 0 \\ 0 & \alpha^{n}\end{array}\right)$ et $u$ est l'endomorphisme $\left(\begin{array}{c}0 \\ t \\ t\end{array} 0^{-1}\right)$. Avec $n \not \equiv n^{2} \equiv 1(\bmod m), m$ de la forme $m=2^{k}(2 l+1)$ et $t \in \mathbb{C}^{\star}$. À difféomorphisme près on peut supposer que $t=1[\mathbf{2 1}]$.

(D2) $H$ est le sous-groupe de $U(2)$ engendré par la matrice $\left(\begin{array}{cc}\alpha & 0 \\ 0 & -\alpha\end{array}\right)$ et $u$ est l'endomorphisme $\left(\begin{array}{cc}0 & t^{-1} \\ t & 0\end{array}\right)$ avec $t \in \mathbb{C}^{\star}$. $\grave{A}$ difféomorphisme près on peut supposer que $t=1[\mathbf{2 1}]$. 
(D3) $H=\langle\alpha \mathrm{Id}\rangle \times B_{2^{k}(2 l+1)}^{\prime}$ et l'endomorphisme $u$ est $\left(\begin{array}{c}0 \\ t \\ t\end{array} t^{-1}\right)$. Avec $\operatorname{pgcd}\left(2^{k}(2 l+1), m\right)=1,2 l+1 \geq 3$ et $k \geq 3$. Enfin $t^{2}=(-\epsilon)^{k}$ et $t \neq 1$.

(D4) $H=\langle\alpha \mathrm{Id}\rangle \times B_{n}$ et l'endomorphisme $u$ est $\left(\begin{array}{cc}e^{\frac{i \pi}{2 n}} & 0 \\ 0 & e^{\frac{-i \pi}{2 n}}\end{array}\right)$. Avec $n \geq 2$ et $\operatorname{pgcd}(m, 4 n)=1$.

(D5) $H=\langle\alpha \mathrm{Id}\rangle \times C$ et l'endomorphisme $u$ est $\left(\begin{array}{cc}e^{\frac{i \pi}{4}} & 0 \\ 0 & e^{\frac{-i \pi}{4}}\end{array}\right)$. Avec $\operatorname{pgcd}(m, 6)=1$.

(D6) $H=\langle\alpha \mathrm{Id}\rangle \times B_{2}$ et l'endomorphisme $u$ est $\frac{1}{\sqrt{2}}\left(\begin{array}{c}e^{\frac{3 i \pi}{4}} e^{\frac{3 i \pi}{4}} \\ e^{\frac{i \pi}{4}}-e^{\frac{i \pi}{4}}\end{array}\right)$. Avec $\operatorname{pgcd}(m, 2)=1$.

2. Les surfaces de Hopf secondaires sont spin : Dans le cas où $G$ est décomposable, la surface de Hopf associée est difféomorphe au produit $\mathbb{S}^{3} / H \times \mathbb{S}^{1}[\mathbf{2 1}]$. Ces surfaces sont parallélisables comme produit d'une 3 -variété compacte orientable avec $\mathbb{S}^{1}$. En particulier elles sont spin. Si $G$ est indécomposable la variété $S=\mathbb{C}^{2 \star} / G$ est la suspension de $u: \mathbb{S}^{3} / H \longrightarrow \mathbb{S}^{3} / H$ au-dessus de $[0,1][\mathbf{2 1}]$.

Dans les cas (D1) et (D2), on se ramène au cas où $t=1$.

On considère alors le sous-groupe de $U(2)$ engendré par $H$ et l'élément $u:\langle H, u\rangle$. Il agit sur la sphère $\mathbb{S}^{3}$. L'action admet un point fixe si et seulement si 1 est valeur propre d'un élément de ce groupe. Mais comme le groupe $\langle H, u\rangle$ est fini et que $H$ est sans point fixe, il existe une racine de l'unité $\xi$ telle que l'action du groupe $K=\langle H, \xi u\rangle$ sur $\mathbb{S}^{3}$ soit propre, discontinue et sans point fixe. Comme toute 3 -variété compacte orientable, la variété $\mathbb{S}^{3} / K$ est parallélisable. C'est-à-dire qu'il existe une base $\left(X_{1}, X_{2}, X_{3}\right)$ de champs de vecteurs sur $\mathbb{S}^{3}$ invariantes par $H$ et $\xi u$. On se fixe une détermination logarithmique définie en $\xi$ et on construit les trois applications suivantes définies sur $[0,1] \times \mathbb{S}^{3}$ et à valeurs dans l'espace tangent à $\mathbb{S}^{3}$ :

$$
\left\{\begin{array}{l}
Y_{1}(t, q)=e^{-t \log \xi} X_{1}\left(e^{t \log \xi} q\right) \\
Y_{2}(t, q)=e^{-t \log \xi} X_{2}\left(e^{t \log \xi} q\right) \\
Y_{3}(t, q)=e^{-t \log \xi} X_{3}\left(e^{t \log \xi} q\right) .
\end{array}\right.
$$

Ces vecteurs sont invariants par le groupe $H$ et par la suspension de $u: \mathbb{S}^{3} / H \longrightarrow \mathbb{S}^{3} / H$ au-dessus de $[0,1]$. Si on note $Y_{4}(t, q)=\frac{\partial}{\partial t}$ le champ de vecteurs constant tangent à la variable $t$. On a une base de champs de vecteurs quelque soit la surface de Hopf secondaire. 
Proposition 3. Les surfaces de Hopf secondaires sont parallélisables. En particulier elles sont spin.

\section{Surfaces d'Inoue.}

1. Les surfaces d'Inoue de type $S_{N}$ sont spin : Soit $N=\left(n_{i j}\right) \in S L(3, \mathbb{Z})$ une matrice dont les valeurs propres sont $\alpha, \beta, \bar{\beta}$ avec $\alpha>1$ et $\beta \neq$ $\bar{\beta}$. Soient $\left(a_{1}, a_{2}, a_{3}\right)$ et $\left(b_{1}, b_{2}, b_{3}\right)$ les vecteurs propres de $N$ associés respectivement à $\alpha$ et $\beta$. On note $\mathbb{H}$ le demi-plan supérieur de $\mathbb{C}$ et $G_{N}$ le groupe d'automorphismes de $\mathbb{H} \times \mathbb{C}$ engendré par :

$$
\left\{\begin{array}{l}
g_{0}:(w, z) \longrightarrow(\alpha w, \beta z) \\
g_{i}:(w, z) \longrightarrow\left(w+a_{i}, z+b_{i}\right) \quad \forall i \in\{1,2,3\} .
\end{array}\right.
$$

Par définition les surfaces $S_{N}=\mathbb{H} \times \mathbb{C} / G_{N}$ sont les surfaces d'Inoue de type neutre [19].

Proposition 4. Les surfaces d'Inoue de type $S_{N}$ sont parallélisables. En particulier elles sont spin.

Preuve: Soit $\left(e_{1}, e_{2}\right)$ la "base" canonique de $\mathbb{H} \simeq \mathbb{R} \oplus i \mathbb{R}^{+}$et $\left(e_{3}, e_{4}\right)$ celle de $\mathbb{C} \simeq \mathbb{R} \oplus i \mathbb{R}$. On considère les quatre champs de vecteurs sur $\mathbb{H} \times \mathbb{C}$ définis par :

$$
\left\{\begin{array}{l}
X_{1}(w, z)=\operatorname{Im} w \cdot e_{1} \\
X_{2}(w, z)=\operatorname{Im} w \cdot e_{2} \\
X_{3}(w, z)=\beta^{\frac{\ln (\operatorname{Im} w)}{\ln \alpha}} \cdot e_{3} \\
X_{4}(w, z)=\beta^{\frac{\ln (\operatorname{Im} w)}{\ln \alpha}} \cdot e_{4} .
\end{array}\right.
$$

Ces quatre champs de vecteurs passent au quotient en une base de champs de vecteurs sur $S_{N}=\mathbb{H} \times \mathbb{C} / G_{N}$.

2. Les surfaces d'Inoue de type $S_{N, p, q, r ; t}^{+}$sont spin : Soit $N=\left(n_{i j}\right) \in$ $S L(2, \mathbb{Z})$ une matrice ayant des valeurs propres réelles $\alpha$ et $1 / \alpha$ avec $\alpha>$ 1 . Soient $\left(a_{1}, a_{2}\right)$ et $\left(b_{1}, b_{2}\right)$ les vecteurs propres de $M$ associés respectivement à $\alpha$ et $1 / \alpha$. On fixe des entiers $p, q, r$ où $r$ est non nul et un nombre complexe $t$. On note $\left(c_{1}, c_{2}\right)$ la solution de l'équation :

$$
\left(c_{1}, c_{2}\right)=\left(c_{1}, c_{2}\right) \cdot{ }^{t} N+\left(e_{1}, e_{2}\right)+\frac{b_{1} a_{2}-b_{2} a_{1}}{r}(p, q)
$$

où $e_{i}=\frac{1}{2} n_{i 1}\left(n_{i 1}-1\right) a_{1} b_{1}+\frac{1}{2} n_{i 2}\left(n_{i 2}-1\right) a_{2} b_{2}+n_{i 1} n_{i 2} b_{1} a_{2} \quad i \in\{1,2\}$. 
On note $G_{N, p, q, r ; t}^{+}$le groupe d'automorphismes de $\mathbb{H} \times \mathbb{C}$ engendré par :

$$
\left\{\begin{array}{l}
g_{0}:(w, z) \longrightarrow(\alpha w, z+t) \\
g_{i}:(w, z) \longrightarrow\left(w+a_{i}, z+b_{i} w+c_{i}\right) \quad \forall i \in\{1,2\} \\
g_{3}:(w, z) \longrightarrow\left(w, z+\frac{b_{1} a_{2}-b_{2} a_{1}}{r}\right) .
\end{array}\right.
$$

Par définition les surfaces $S_{N, p, q, r ; t}^{+}=\mathbb{H} \times \mathbb{C} / G_{N, p, q, r ; t}^{+}$sont les surfaces d'Inoue de type positif [19].

Proposition 5. Les surfaces d'Inoue de type $S_{N, p, q, r ; t}^{+}$sont parallélisables. En particulier elles sont spin.

Preuve: On note encore $\left(e_{1}, e_{2}\right)$ la "base" canonique de $\mathbb{H} \simeq \mathbb{R} \oplus i \mathbb{R}^{+}$ et $\left(e_{3}, e_{4}\right)$ celle de $\mathbb{C} \simeq \mathbb{R} \oplus i \mathbb{R}$. On considère les deux champs de vecteurs sur $\mathbb{H} \times \mathbb{C}$ définis par :

$$
\left\{\begin{array}{l}
X_{3}(w, z)=e_{3} \\
X_{4}(w, z)=e_{4} .
\end{array}\right.
$$

Ces deux champs passent au quotient sur $S_{N, p, q, r ; t}^{+}=\mathbb{H} \times \mathbb{C} / G_{N, p, q, r ; t}^{+}$. On définit sur $\mathbb{H} \times \mathbb{C}$ le champ de 2-plans Vect $\left(e_{1}, e_{3}\right)$ orientés par le bivecteur $e_{1} \wedge e_{3}$. Ce champ passe au quotient en un champ de 2-plans orientés sur $S_{N, p, q, r ; t}^{+}$noté $P_{13}$. On munit $S_{N, p, q, r ; t}^{+}$d'une métrique riemannienne et on note $X_{1}$ le champ de vecteurs dans $P_{13}$ unitaire et tel que $\left(X_{1}, X_{3}\right)$ soit une famille orthogonale directe de $P_{13}$.

De même le champ de 2-plans $\operatorname{Vect}\left(e_{2}, e_{4}\right)$ constant sur $\mathbb{H} \times \mathbb{C}$ et orientés par le bivecteur $e_{2} \wedge e_{4}$ passe au quotient sur $S_{N, p, q, r ; t}^{+}$en un champ de 2-plans $P_{24}$ orientés. On définit de même le champ de vecteurs $X_{2}$ dans $P_{24}$ unitaire et tel que $\left(X_{2}, X_{4}\right)$ soit une famille orthogonale directe de $P_{24}$.

On a ainsi défini une base de champs de vecteurs $\left(X_{1}, X_{2}, X_{3}, X_{4}\right)$ sur $S_{N, p, q, r ; t}^{+}$. Les surfaces d'Inoue de type positive sont donc bien parallélisables.

3. Les surfaces d'Inoue de type $S_{N, p, q, r}^{-}$sont spin : Soit $N=\left(n_{i j}\right) \in$ $G L(2, \mathbb{Z})$ une matrice de détermiant -1 ayant deux valeurs propres réelles $\alpha,-1 / \alpha$ avec $\alpha>1$. Soient $\left(a_{1}, a_{2}\right)$ et $\left(b_{1}, b_{2}\right)$ les vecteurs propres de $M$ associés respectivement à $\alpha$ et $-1 / \alpha$. Comme pour les surfaces d'Inoue de type positive, on fixe des entiers $p, q, r$ où $r$ est non nul et on note $\left(c_{1}, c_{2}\right)$ la solution de l'équation :

$$
-\left(c_{1}, c_{2}\right)=\left(c_{1}, c_{2}\right) \cdot{ }^{t} N+\left(e_{1}, e_{2}\right)+\frac{b_{1} a_{2}-b_{2} a_{1}}{r}(p, q)
$$

où $e_{i}=\frac{1}{2} n_{i 1}\left(n_{i 1}-1\right) a_{1} b_{1}+\frac{1}{2} n_{i 2}\left(n_{i 2}-1\right) a_{2} b_{2}+n_{i 1} n_{i 2} b_{1} a_{2} \quad i \in\{1,2\}$. 
On note $G_{N, p, q, r}^{-}$le groupe d'automorphismes de $\mathbb{H} \times \mathbb{C}$ engendré par :

$$
\left\{\begin{array}{l}
g_{0}:(w, z) \longrightarrow(\alpha w,-z) \\
g_{i}:(w, z) \longrightarrow\left(w+a_{i}, z+b_{i} w+c_{i}\right) \quad \forall i \in\{1,2\} \\
g_{3}:(w, z) \longrightarrow\left(w, z+\frac{b_{1} a_{2}-b_{2} a_{1}}{r}\right) .
\end{array}\right.
$$

Par définition les surfaces $S_{N, p, q, r}^{-}=\mathbb{H} \times \mathbb{C} / G_{N, p, q, r}^{-}$sont les surfaces d'Inoue de type négatif [19].

Proposition 6. Les surfaces d'Inoue de type $S_{N, p, q, r}^{-}$sont parallélisables. En particulier elles sont spin.

Preuve: On garde les notations précédentes. On définit sur $\mathbb{H} \times \mathbb{C}$ le champ constant de 3 -plans $\operatorname{Vect}\left(e_{1}, e_{3}, e_{4}\right)$ orientés grâce au trivecteur $e_{1} \wedge e_{3} \wedge e_{4}$. Ce champ passe au quotient sur $S_{N, p, q, r}^{-}$en un champ de 3-plans orientés noté $P_{134}$.

Pour tout vecteur $X$ de $\mathbb{C} \simeq \operatorname{Vect}\left(e_{3}, e_{4}\right)$, on note $R_{\theta}(X)$ la rotation d'angle $\theta$ du vecteur $X$ dans le plan Vect $\left(e_{3}, e_{4}\right)$ orientée par $e_{3} \wedge e_{4}$. Sur $\mathbb{H} \times \mathbb{C}$ on considère les deux champs de vecteurs définis par :

$$
\left\{\begin{array}{l}
X_{3}(w, z)=R_{\frac{\ln (\operatorname{Im} w)}{\ln \alpha} \pi}\left(e_{3}\right) \\
X_{4}(w, z)=R_{\frac{\ln (\operatorname{Im} w)}{\ln \alpha} \pi}\left(e_{4}\right) .
\end{array}\right.
$$

Ces vecteurs passent au quotient en deux champs de vecteurs sur $S_{N, p, q, r}^{-}=\mathbb{H} \times \mathbb{C} / G_{N, p, q, r}^{-}$. On munit $S_{N, p, q, r}^{-}$d'une métrique riemannienne. On peut alors définir $X_{1}$ le champ unitaire de $P_{134}$ tel que $X_{1}$ soit orthogonal à $\operatorname{Vect}\left(X_{3}, X_{4}\right)$ et tel que $\left(X_{1}, X_{3}, X_{4}\right)$ soit une famille orientée de $P_{134}$. Enfin on note $X_{2}$ le champ de vecteurs unitaire orthogonal à $P_{134}$ tel que $\left(X_{1}, X_{2}, X_{3}, X_{4}\right)$ soit une famille orientée.

4. Remarque sur la parallélisabilité des surfaces d'Inoue : On sait depuis l'article de Wall [35] (lire aussi [3], [24]) qu'à revêtement fini près les surfaces d'Inoue sont toutes des solvsurfaces affines, c'est-à-dire des quotients de la forme $G / \Gamma$, où $G$ est un sous-groupe résoluble du groupe affine et $\Gamma$ un réseau cocompact de $G$. Comme une base de champs de vecteurs invariants à gauche sur $G$ descend en une base de champs de vecteurs sur $G / \Gamma$, toute solvsurface affine est parallélisable. On obtient ainsi directement la parallélisabilité des surfaces d'Inoue de type $S_{N, p, q, r ; t}^{+}$ pour lesquelles le paramètre $t$ n'est pas réel. Mais ce raisonnement ne marche pas pour les autres surfaces qui ne sont que des quotients finis de solvsurfaces affines. 


\section{Surfaces hyperelliptiques.}

Pour les surfaces hyperelliptiques, on a encore une description explicite $\left[\mathbf{2}\right.$, Chapter V.5]. Ainsi soit $\mathbb{T}_{1}$ le tore $\mathbb{C} / \mathbb{Z}+i \mathbb{Z}$ et $\mathbb{T}_{2}$ le tore $\mathbb{C} / \Gamma$, où $\Gamma$ est un réseau de $\mathbb{C}$. On note $z_{1}$ et $z_{2}$ les coordonnées complexes sur $\mathbb{T}_{1}$ et $\mathbb{T}_{2}$ respectivement. À difféomorphisme près une surface hyperelliptique est le quotient de $\mathbb{T}_{1} \times \mathbb{T}_{2}$ par un des groupes d'automorphismes $G$ suivant :

\begin{tabular}{|c|c|c|c|}
\hline Type & $\boldsymbol{\Gamma}$ & $\boldsymbol{G}$ & Action de $\boldsymbol{G}$ sur $\mathbb{T}_{\mathbf{1}} \times \mathbb{T}_{\mathbf{2}}$ \\
\hline 1 & $\mathbb{Z}+i \mathbb{Z}$ & $\mathbb{Z} / 2 \mathbb{Z}$ & $g_{1}\left(z_{1}, z_{2}\right)=\left(z_{1}+\frac{1}{2},-z_{2}\right)$ \\
2 & $\mathbb{Z}+i \mathbb{Z}$ & $\mathbb{Z} / 2 \mathbb{Z} \oplus \mathbb{Z} / 2 \mathbb{Z}$ & $\left\{\begin{array}{l}g_{1}\left(z_{1}, z_{2}\right)=\left(z_{1}+\frac{1}{2},-z_{2}\right) \\
g_{2}\left(z_{1}, z_{2}\right)=\left(z_{1}+\frac{i}{2}, z_{2}+e_{1}\right) \quad \text { avec } 2 e_{1}=0\end{array}\right.$ \\
\hline 3 & $\mathbb{Z}+j \mathbb{Z}$ & $\mathbb{Z} / 3 \mathbb{Z}$ & $g_{1}\left(z_{1}, z_{2}\right)=\left(z_{1}+\frac{1}{3}, j z_{2}\right)$ \\
4 & $\mathbb{Z}+j \mathbb{Z}$ & $\mathbb{Z} / 3 \mathbb{Z} \oplus \mathbb{Z} / 3 \mathbb{Z}$ & $\left\{\begin{array}{c}g_{1}\left(z_{1}, z_{2}\right)=\left(z_{1}+\frac{1}{3}, j z_{2}\right) \\
g_{2}\left(z_{1}, z_{2}\right)=\left(z_{1}+\frac{i}{3}, z_{2}+e_{1}\right) \quad \text { avec } j e_{1}=e_{1}\end{array}\right.$ \\
\hline 5 & $\mathbb{Z}+i \mathbb{Z}$ & $\mathbb{Z} / 4 \mathbb{Z}$ & $g_{1}\left(z_{1}, z_{2}\right)=\left(z_{1}+\frac{1}{4}, i z_{2}\right)$ \\
6 & $\mathbb{Z}+i \mathbb{Z}$ & $\mathbb{Z} / 4 \mathbb{Z} \oplus \mathbb{Z} / 2 \mathbb{Z}$ & $\left\{\begin{array}{l}g_{1}\left(z_{1}, z_{2}\right)=\left(z_{1}+\frac{1}{4}, i z_{2}\right) \\
g_{2}\left(z_{1}, z_{2}\right)=\left(z_{1}+\frac{i}{2}, z_{2}+e_{1}\right) \quad \text { avec } i e_{1}=e_{1}\end{array}\right.$ \\
\hline 7 & $\mathbb{Z}+j \mathbb{Z}$ & $\mathbb{Z} / 6 \mathbb{Z}$ & $g_{1}\left(z_{1}, z_{2}\right)=\left(z_{1}-\frac{1}{6},-j z_{2}\right)$ \\
\hline
\end{tabular}

Comme pour les surfaces d'Inoue, on construit explicitement une base de champs de vecteurs. Le premier tore $\mathbb{T}_{1}$ est le quotient $\mathbb{C} / \mathbb{Z}+i \mathbb{Z}$. On note $\left(e_{1}, e_{2}\right)$ la base canonique de $\mathbb{C} \simeq \mathbb{R}+i \mathbb{R}$. Le deuxième tore est le quotient $\mathbb{T}_{2}=\mathbb{C} / \Gamma$. On note ici $\left(e_{3}, e_{4}\right)$ la base canonique de $\mathbb{C} \simeq \mathbb{R}+i \mathbb{R}$ et $R_{\theta}$ la rotation d'angle $\theta$ dans le plan $\mathbb{C} \simeq \operatorname{Vect}\left(e_{3}, e_{4}\right)$ orienté par $e_{3} \wedge e_{4}$. On définit sur $\mathbb{C} \oplus \mathbb{C}$ les quatre champs de vecteurs :

$$
\left\{\begin{array}{l}
X_{1}\left(z_{1}, z_{2}\right)=e_{1} \\
X_{2}\left(z_{1}, z_{2}\right)=e_{2} \\
X_{3}\left(z_{1}, z_{2}\right)=R_{2 \pi \operatorname{Re} z_{1}}\left(e_{3}\right) \\
X_{4}\left(z_{1}, z_{2}\right)=R_{2 \pi \operatorname{Re} z_{1}}\left(e_{4}\right) .
\end{array}\right.
$$

Ces champs passent naturellement au quotient en quatre champs sur $\mathbb{T}_{1} \times \mathbb{T}_{2}$, quelque soit le réseau $\Gamma$ définissant $\mathbb{T}_{2}=\mathbb{C} / \Gamma$. De plus quelque soit le type de la surface hyperelliptique, ces champs passent au quotient 
sur $\mathbb{T}_{1} \times \mathbb{T}_{2} / G$. On a ainsi construit une base de champs de vecteurs sur chaque surface hyperelliptique.

Proposition 7. Les surfaces hyperelliptiques sont parallélisables. En particulier elles sont spin.

\section{3. Étude et résolution de notre problème}

On rappelle que notre but est de classifier l'ensemble des 4-variétés riemanniennes orientées $(M, g)$ telle que :

(i) $M$ admet une structure complexe,

(ii) la fibration twistorielle de $(M, g)$ est $\mathcal{C}^{\infty}$-triviale,

(iii) la métrique $g$ est anti-autoduale.

Pour de telles variétés on a deux structures complexes sur le produit $M \times \mathbb{S}^{2}$ : la structure complexe produit et celle d'espace twistoriel. Cette dernière n'est jamais biméromorphe à une structure kählerienne $[\mathbf{1 1}]$.

Sous les hypothèses de notre problème on a le résultat suivant :

Proposition 8. La structure presque complexe d'espace twistoriel n'est jamais une déformation de la structure complexe produit.

Preuve: Comme $(M, g)$ est presque hypercomplexe, son espace twistoriel $Z$ est difféomorphe au produit $M \times \mathbb{S}^{2}$. Son deuxième groupe de cohomologie de De Rham est donc : $H^{2}(Z, \mathbb{Z})=H^{2}(M, \mathbb{Z}) \oplus H^{2}\left(\mathbb{S}^{2}, \mathbb{Z}\right)$. On note $h$ le générateur du $H^{2}\left(\mathbb{S}^{2}, \mathbb{Z}\right) \simeq \mathbb{Z}$.

L'espace twistoriel de $(M, g)$ est muni d'une structure presque complexe canonique $J_{e t}$ dont la première classes de Chern notée $c_{1}\left(J_{e t}\right)$ est égale à $4 h[\mathbf{1 8}]$. On note $c_{1}\left(J_{p}\right)$ la première classe de Chern de la variété $M \times \mathbb{S}^{2}$ munie de la structure complexe produit et $c_{1}(M)$ celle de $M$. On a $c_{1}\left(J_{p}\right)=2 h+c_{1}(M)$. Les classes de Chern étant différentes, la structure d'espace twistoriel n'est donc jamais une déformation de la structure produit.

\subsection{Résolution du problème.}

Le théorème suivant donne la liste des surfaces presque hypercomplexes qui peuvent être munies d'une métrique riemannienne anti-autoduale. 
Théorème 2. Soit $M$ une surface compacte complexe dont le fibré twistoriel est $\mathcal{C}^{\infty}$-trivial. Si la variété $M$ peut être munie d'une métrique riemannienne anti-autoduale alors $M$ est:

a) une surface $K 3$,

b) un tore,

c) une surface hyperelliptique,

d) une surface de Hopf,

e) une surface minimale spin de dimension de Kodaira un.

Réciproquement dans les cas a), b), c) et d) il existe toujours une métrique riemannienne anti-autoduale.

Les seules variétés non connues susceptibles d'admettre une métrique anti-autoduale sont les surfaces proprement elliptiques. Ces surfaces se divisent en deux groupes suivant que la caractéristique d'Euler est nulle ou pas. Dans les deux cas on peut préciser un peu le théorème. Par le théorème de Schoen [32] toute métrique anti-autoduale est conforme à une métrique dont la courbure scalaire est constante. Pour les deux remarques qui suivent, on supposera donc que la courbure scalaire de la métrique anti-autoduale est constante.

Remarque. Soit $M$ une surface minimale de dimension de Kodaira un spin et de caractéristique d'Euler nulle. Si $M$ admet une métrique $g$ anti-autoduale alors la métrique est localement conformément plate, à courbure scalaire (strictement) négative $[\mathbf{7}]$, non hermitienne $[\mathbf{8}]$, non Einstein et à holonomie sans sous-espace invariant $[\mathbf{2 6}]$. En particulier la métrique n'est pas Ricci parallèle.

Remarque. Soit $M$ une surface minimale de dimension de Kodaira un spin et de caractéristique d'Euler $\chi>0$. Si $M$ admet une métrique anti-autoduale alors la métrique est à courbure scalaire (strictement) négative, non Einstein [17] (et donc à courbure non harmonique [7]), non localement conformément plate, non hermitienne [8], et à holonomie sans sous-espace invariant. Là encore la métrique n'est pas Ricci parallèle.

\subsection{Démonstration.}

1. Sens direct du Théorème 2 : Pour démontrer le Théorème 2 on étudie au cas par cas les différentes surfaces complexes à fibré twistoriel trivial. Lorsque la signature d'une variété est nulle, les métriques antiautoduales sont localement conformément plates [4]. On peut alors utiliser un résultat de Goldman et Kamishima. On rappelle qu'un groupe est dit virtuellement résoluble s'il admet un sous-groupe résoluble d'indice fini. 
Théorème $([\mathbf{1 6}],[\mathbf{2 0}])$. Si $(M, g)$ est une 4-variété compacte localement conformément plate à groupe fondamental virtuellement résoluble alors à revêtement riemannien fini près $M$ est conforme à la sphère ronde $\mathbb{S}^{4}$, à un tore plat $\mathbb{T}^{4}$ ou au produit $\mathbb{S}^{1} \times \mathbb{S}^{3}$.

Surfaces d'Inoue. Si une surface d'Inoue possédait une métrique antiautoduale, elle serait localement conformément plate car sa signature est nulle [19]. De plus le groupe fondamental d'une surface d'Inoue est résoluble $[\mathbf{1 9}]$. Comme les surfaces d'Inoue ne sont recouvertes ni par $\mathbb{S}^{4}$ ni par $\mathbb{S}^{1} \times \mathbb{S}^{3}$ ni par $\mathbb{T}^{4}$, par le théorème de Goldman et Kamishima on en déduit qu'elles n'admettent pas de métrique anti-autoduale.

Surfaces réglées de genre un. Soit $S$ une surface réglée de genre un admettant une structure spin. À difféomorphisme près $S$ est égale au produit $\mathbb{S}^{2} \times \mathbb{T}^{2}[\mathbf{2}],[\mathbf{1 5}]$. On en déduit qu'une surface réglée de genre un spin n'admet aucune métrique localement conformément plate, ni antiautoduale.

Surfaces de Kodaira. Le revêtement universel d'une surface de Kodaira primaire est $\mathbb{C}^{2}$. Son groupe fondamental est isomorphe au groupe engendré par quatre éléments de la forme [25] :

$$
\begin{cases}g_{i}(z, w)=\left(z, w+\beta_{i}\right) & i=1,2 \\ g_{i}(z, w)=\left(z+\alpha_{i}, w+\bar{\alpha}_{i} z+\beta_{i}\right) & i=3,4\end{cases}
$$

et soumis à la relation : $g_{3} g_{4}=g_{1}^{m} g_{4} g_{3}, m \in \mathbb{N}^{\star}$. Par conséquent le groupe fondamental d'une surface de Kodaira primaire est nilpotent et celui d'une surface de Kodaira secondaire virtuellement nilpotent.

Si une surface de Kodaira possédait une métrique anti-autoduale, elle serait localement conformément plate $(\tau=0)$. Comme le groupe fondamental d'une surface de Kodaira est virtuellement nilpotent, le théorème de Goldman et Kamishima nous assure qu'elle n'admet pas de métrique anti-autoduale.

2. Réciproque du Théorème 3 : Les tores et les surfaces de Hopf primaires (i.e. difféomorphes à $\mathbb{S}^{1} \times \mathbb{S}^{3}$ ) admettent une métrique riemannienne localement conformément plate. De plus la métrique de Calabi-Yau sur une surface $K 3$ est anti-autoduale (car kählerienne à courbure scalaire nulle). Pour les surfaces hyperelliptiques, on a donné une description explicite à difféomorphisme près de ces surfaces et il est facile de voir que la métrique plate sur $\mathbb{R}^{4}$ descend en une métrique plate sur ces surfaces. Comme la signature d'une surface de Hopf est nulle, la seule chose qu'il 
reste à montrer est donc que toute surface de Hopf secondaire admet une métrique riemannienne localement conformément plate.

Une surface de Hopf secondaire est le quotient de $\mathbb{C}^{2 \star}$ par un groupe $G$. À difféomorphisme près on peut toujours supposer que $G$ est un sousgroupe de $G L\left(\mathbb{C}^{2}\right)[\mathbf{2 1}]$. Nous allons voir qu'on peut toujours se ramener au cas où $G$ est un sous-groupe du groupe des isométries de $\mathbb{R}^{4 \star}$ muni de la métrique :

$$
V=\frac{d x_{1}^{2}+d x_{2}^{2}+d x_{3}^{2}+d x_{4}^{2}}{x_{1}^{2}+x_{2}^{2}+x_{3}^{2}+x_{4}^{2}} .
$$

Si bien que cette métrique descend en une métrique localement conformément plate sur $\mathbb{R}^{4 \star} / G$. Soit $(m, n) \in \mathbb{N}^{\star}$ deux entiers, $\alpha$ une racine primitive $m^{\text {ième }}$ de l'unité et $(\lambda, \alpha, \beta) \in \mathbb{C}$ tels que $0<|\alpha| \leq|\beta|<1$. On rappelle ici quelques résultats de Kato $[\mathbf{2 1}],[\mathbf{2 3}]$ :

(1) Si $G$ est décomposable et abélien alors :

(A) Soit $G$ est engendré par les applications :

$g\left(z_{1}, z_{2}\right)=\left(\alpha z_{1}, \beta z_{2}\right)$ et $h\left(z_{1}, z_{2}\right)=\left(a z_{1}, a^{n} z_{2}\right)$ avec $\operatorname{pgcd}(m, n)=1$.

(B) Soit $G$ est engendré par les applications :

$$
g\left(z_{1}, z_{2}\right)=\left(\alpha z_{1}+\lambda z_{2}, \alpha z_{2}\right) \text { et } h\left(z_{1}, z_{2}\right)=\left(a z_{1}, a z_{2}\right) .
$$

C'est-à-dire que $G$ n'est pas un groupe d'isométries. Mais quitte à faire une petite déformation, on peut se ramener à l'un des deux cas suivant :

(A') $G$ est engendré par les applications :

$g\left(z_{1}, z_{2}\right)=\left(\alpha z_{1}, \beta z_{2}\right)$ et $h\left(z_{1}, z_{2}\right)=\left(a z_{1}, a^{n} z_{2}\right)$ avec $|\alpha|=|\beta|$.

(B') $G$ est engendré par les applications :

$$
g\left(z_{1}, z_{2}\right)=\left(\alpha z_{1}, \alpha z_{2}\right) \text { et } h\left(z_{1}, z_{2}\right)=\left(a z_{1}, a z_{2}\right) .
$$

(2) $\mathrm{Si} G$ est décomposable mais non abélien, le groupe $G$ est un groupe d'isométries de $\left(\mathbb{R}^{4 \star}, V\right)$.

(3) Si $G$ est indécomposable, dans les cas (D3)-(D6) (cf. Partie 3.1) $G$ est un groupe d'isométries. Et dans les deux premiers cas, à difféomorphisme près, on peut se ramener au cas où le paramètre $t$ de l'endomorphisme $u$ est égale à 1 . Et alors la métrique $V$ de $\mathbb{R}^{4 \star}$ passe au quotient.

Conclusion. Toutes les surfaces de Hopf secondaires possèdent une métrique riemannienne localement conformément plate. 


\section{Application}

En utilisant le Théorème 2, on peut donner une famille d'exemples de 4 -variétés $M$ sans structure complexe dont le produit $M \times \mathbb{C} P^{1}$ est complexe. C'est en quelque sorte une structure complexe "non standard".

Théorème 3. Pour toute 3 -variété compacte hyperbolique orientable $H$, il existe une structure complexe sur le produit $H \times \mathbb{S}^{1} \times \mathbb{S}^{2}$ bien que $H \times \mathbb{S}^{1}$ n'en possède pas.

Ce théorème fournit en particulier une famille d'exemples simples de 4 -variétés parallélisables mais qui ne possèdent pas de structure complexe. De tels exemples existaient déjà $[\mathbf{3 7}],[\mathbf{1 0}]$ mais restaient assez isolés ou peu explicites.

Corollaire. Pour toute 3-variété compacte hyperbolique orientable $H$, le produit $H \times \mathbb{S}^{1}$ est une 4-variété parallélisable sans structure complexe.

Remarque. La démonstration que nous donnons du Théorème 3 permet également de montrer que tout fibré en cercle $\mathbb{S}^{1}$ au-dessus d'une 3 -variété hyperbolique compacte orientable $H$ est parallélisable sans structure complexe.

Preuve du Théorème 3: Si $H$ est une 3-variété hyperbolique orientable compacte, elle est parallélisable donc $H \times \mathbb{S}^{1}$ aussi. Son espace twistoriel est donc difféomorphe à $H \times \mathbb{S}^{1} \times \mathbb{S}^{2}$. De plus on peut toujours munir $H$ d'une métrique hyperbolique c'est-à-dire d'une métrique à courbure sectionnelle constante égale à -1 . La métrique produit sur $H \times \mathbb{S}^{1}$ est alors localement conformément plate [4, Chapter $1-\mathrm{J}]$ et l'espace twistoriel associé à cette variété riemannienne orientée admet une structure complexe [1].

Reste à montrer que le produit $H \times \mathbb{S}^{1}$ n'admet aucune structure complexe. Pour cela on utilise le Théorème 2 et les propriétés du groupe fondamental d'une variété hyperbolique compacte. Par les théorèmes de Preissman et de Byers [4, Chapter 6 -C] on sait que le groupe fondamental de $H$, noté $\pi_{1}(H)$, n'est pas abélien et que les seuls sous-groupes résolubles non nuls de $\pi_{1}(H)$ sont infinis cycliques. En particulier il n'est pas résoluble et n'admet pas de sous-groupe isomorphe à $\mathbb{Z}^{2}$.

Si $H \times \mathbb{S}^{1}$ admet une structure complexe alors c'est une des surfaces du Théorème 2, le seul cas à éliminer est le cas des surfaces elliptiques de dimension de Kodaira 0 ou 1 . Si $X$ est une surface elliptique elle admet une surjection holomorphe $\pi: X \longrightarrow C$ dont la fibre générique est une courbe elliptique. Si $X$ est parallélisable et de dimension de Kodaira 0 ou 1 alors $X$ admet un revêtement fini $\tilde{X} \longrightarrow X$ où $\tilde{X}$ est une surface 
elliptique au-dessus d'une surface de Riemann de genre $g \geq 1$, sans fibre multiple et tel que le groupe fondamental de la fibre $\left(\mathbb{Z}^{2}\right)$ est central dans celui de $\tilde{X}[\mathbf{2}]$. Le groupe fondamental de $\tilde{X}$ admet donc un sous-groupe isomorphe à $\mathbb{Z}^{3}$.

Si $H \times \mathbb{S}^{1}$ était munie d'une structure complexe, ce serait une surface minimale de dimension de Kodaira 0 ou 1. Et donc $H \times \mathbb{S}^{1}$ admettrait $\tilde{X}$ comme revêtement fini. Son groupe fondamental contiendrait un $\mathbb{Z}^{3}$. Ce qui est impossible puisque le groupe fondamental de $H$ ne contient pas de $\mathbb{Z}^{2}$.

\section{Existence d'une infinité de structures complexes non standards}

On dit qu'une 4-variété riemannienne orientée $(M, g)$ est hypercomplexe s'il existe trois structures complexes $I, J$ et $K$ compatibles avec l'orientation et la métrique et telles que $I J=-J I=K$. La métrique $g$ est alors anti-autoduale [31]. De plus ces trois structures complexes définissent un difféomorphisme $\tau(M, g) \simeq M \times \mathbb{S}^{2}$ tel que la projection sur le deuxième facteur soit une submersion holomorphe de $\tau(M, g)$ sur $\mathbb{C} P^{1}[\mathbf{9}]$. La distribution $T M$ (donnée par ce difféomorphisme) est donc invariante par la structure complexe canonique sur $\tau(M, g)$.

Théorème $([\mathbf{9}])$. Si $(M, g)$ est une 4-variété compacte hypercomplexe alors elle est conforme $\grave{a}$ :

a) un tore plat,

b) une surface K3 munit de la métrique de Calabi-Yau,

c) une surface de Hopf quaternionique (cf. [22]).

Proposition 9. Soit $(M, g)$ une variété hypercomplexe. Il existe une infinité de structures complexes sur les produits $M \times \mathbb{S}^{2}$ qui ne sont pas déformations les unes des autres.

La démonstration de cette proposition s'inspire fortement d'un résultat de LeBrun [27] établi pour les surfaces $K 3$.

Preuve: Pour simplifier l'écriture on notera $\pi: Z \longrightarrow M$ l'espace twistoriel de $(M, g)$. Comme $(M, g)$ est hypercomplexe son espace twistoriel admet une submersion holomorphe sur $\mathbb{C} P^{1}$ :

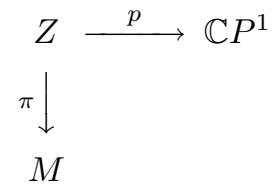


Soit $d \in \mathbb{N}$ un entier et $f_{d}$ l'application holomorphe de degré $d$ définie par :

$$
\begin{aligned}
f_{d}: \quad \mathbb{C} P^{1} & \longrightarrow \mathbb{C} P^{1} \\
{\left[z_{1}, z_{2}\right] } & \longmapsto\left[z_{1}^{d}, z_{2}^{d}\right] .
\end{aligned}
$$

On note $Z_{d}=f_{d}^{\star} Z$ le fibré tiré en arrière :

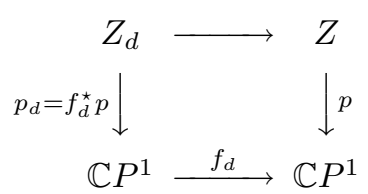

Comme le fibré $p: Z \longrightarrow \mathbb{C} P^{1}$ est $\mathcal{C}^{\infty}$-trivial, il en va de même pour $p_{d}: Z_{d} \longrightarrow \mathbb{C} P^{1}$. Si bien qu'on peut identifier la variété $Z_{d}$ avec la variété $M \times \mathbb{S}^{2}$ munie d'une structure presque complexe intégrable, que l'on notera $J_{d}$. Notons qu'avec ces notations $J_{0}$ est une structure complexe produit et $J_{1}$ est la structure complexe d'espace twistoriel sur $M \times \mathbb{S}^{2}$. Pour montrer que ces différentes structures complexes ne sont pas déformations les unes des autres il suffit de calculer les classes de Chern associées à ces différentes structures [18], [27].

Dans le cas où $M$ est un tore plat ou une surface $K 3$ munie de la métrique de Calabi-Yau, la structure complexe produit sur $M \times \mathbb{C} P^{1}$ est kählerienne. En reprenant la démonstration d'Hitchin [18] il n'est pas très difficile de voir qu'en dehors de ces deux cas, les 3-variétés complexes que nous venons d'introduire ne sont jamais biméromorphes à une variété kählerienne.

Proposition 10. Soit $(M, g)$ une 4-variété hypercomplexe. Quelque soit l'entier d non nul la variété complexe $\left(M \times \mathbb{S}^{2}, J_{d}\right)$ n'est pas biméromorphe à une variété kählerienne.

Preuve: Une variété biméromorphe à une variété kählerienne admet une décomposition de Hodge [13, Théorème 12.9]. On parle de variétés dans la classe $(\mathcal{C})$ de Fujiki. Si $M$ est une surface de Hopf, le premier nombre de Betti de la variété $M \times \mathbb{S}^{2}$ est 1 : aucune des structures complexes $J_{d}$ ne peut être biméromorphe à une structure kählerienne. On se limite donc à l'étude du cas où $M$ est un tore ou une surface $K 3$. Commençons par montrer le lemme suivant :

Lemme. Soit $p$ et d deux entiers différents de zéro. Il n'existe pas de p-forme holomorphe sur $Z_{d}$ non nulle. 
Preuve: On note $\pi_{d}$ la projection de $Z_{d} \simeq M \times \mathbb{S}^{2}$ sur $M$ :

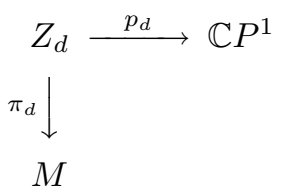

Et pour alléger les notations, on pose $N_{d}=\pi_{d}^{\star} T M$ et $T_{d}=p_{d}^{\star} T \mathbb{S}^{2}$. Soit $\bigwedge^{p} T^{\star} Z_{d}$ le fibré des $p$-formes holomorphes sur $Z_{d}$. On se fixe une fibre $\pi_{d}^{-1}(m)$ et on restreint les fibrés $\bigwedge^{p} T^{\star} Z_{d}, N_{d}$ et $T_{d}$ à cette fibre. La décomposition $T Z_{d}=N_{d} \oplus T_{d}$ induit la suite exacte :

$$
0 \longrightarrow \bigwedge^{p} N_{d}^{\star} \longrightarrow \bigwedge^{p} T^{\star} Z_{d} \longrightarrow T_{d}^{\star} \otimes \bigwedge^{p-1} N_{d}^{\star} \longrightarrow 0 .
$$

Or au-dessus de cette fibre $T_{d}$ et $N_{d}$ sont précisément le fibré tangent et le fibré normal de $\pi_{d}^{-1}(m)$ dans $Z_{d}$. On a donc d'une part $T_{d}^{\star}=\mathcal{O}(-2)$ et d'autre part $N_{d}=\mathcal{O}(d) \oplus \mathcal{O}(d)$. Pour $p>0$ les fibrés $\wedge^{p} N_{d}^{\star}$ et $T_{d}^{\star} \otimes \bigwedge^{p-1} N_{d}^{\star}$ sont des fibrés négatifs. Une section holomorphe globale de $\bigwedge^{p} T^{\star} Z_{d} \longrightarrow Z_{d}$ s'annule sur la fibre $\pi_{d}^{-1}(m)$ pour tout choix de $m \in M$ : elle est identiquement nulle.

Pour finir la preuve de la Proposition 10 on note $\Omega^{p}$ le faisceau des germes de $p$-formes holomorphes sur $Z_{d}$ et $h^{p, q}=\operatorname{dim} H^{q}\left(Z_{d}, \Omega^{p}\right)$. Si $Z_{d}$ est dans la classe $(\mathcal{C})$ de Fijiki, la théorie de Hodge nous donne $h^{p, q}=$ $h^{q, p}$. Si $d$ est non nul alors le lemme ci-dessus nous assure que le genre arithmétique défini par $1-h^{0,1}+h^{0,2}-h^{0,3}$ est égale à 1 . Et d'après le théorème de Riemann-Roch il est aussi égale à $\frac{c_{1} c_{2}}{24}$. Si la variété $Z_{d}$ est biméromorphe à une variété kählerienne on a donc :

$$
\frac{c_{1} c_{2}}{24}=\frac{2(d+1) \chi}{24}=1 .
$$

Cette relation n'est pas compatible avec le fait que $M$ soit une surface $K 3$ $(\chi=24)$ ou un tore $(\chi=0)$ : si $d$ est un entier non nul $Z_{d}$ n'est jamais biméromorphe à une variété kählerienne.

Remarque. Soit $\Sigma_{g}$ une surface de Riemann de genre $g \geq 1$ et $M$ une 4 -variété hypercomplexe. En considérant des applications holomorphes $f: \Sigma_{g} \longrightarrow \mathbb{C} P^{1}$ on peut de la même manière construire une infinité de structures complexes sur $M \times \Sigma_{g}$ qui ne sont ni déformations les unes des autres ni biméromorphes à une structure Kähler. 
Remerciements. Un grand merci à Massimiliano Pontecorvo, Paul Gauduchon et Andrei Teleman pour les dicussions que j'ai pu avoir avec eux. Un grand merci également à Laurent Meerssemann pour son suivi pendant ma thèse.

\section{Références}

[1] M. F. Atiyah, N. J. Hitchin, et I. M. Singer, Self-duality in four-dimensional Riemannian geometry, Proc. Roy. Soc. London Ser. A 362(1711) (1978), 425-461.

[2] W. P. Barth, K. Hulek, C. A. M. Peters, et A. Van de Ven, "Compact complex surfaces", Second edition, Ergebnisse der Mathematik und ihrer Grenzgebiete 3. Folge. A Series of Modern Surveys in Mathematics [Results in Mathematics and Related Areas. 3rd Series. A Series of Modern Surveys in Mathematics] 4, SpringerVerlag, Berlin, 2004.

[3] F. A. Belgun, On the metric structure of non-Kähler complex surfaces, Math. Ann. 317(1) (2000), 1-40.

[4] A. L. Besse, "Einstein manifolds", Ergebnisse der Mathematik und ihrer Grenzgebiete (3) [Results in Mathematics and Related Areas (3)] 10, Springer-Verlag, Berlin, 1987.

[5] A. Blanchard, Sur les variétés analytiques complexes, Ann. Sci. Ecole Norm. Sup. (3) 73 (1956), 157-202.

[6] F. A. Bogomolov, Surfaces of class $\mathrm{VII}_{0}$ and affine geometry, Izv. Akad. Nauk SSSR Ser. Mat. 46(4) (1982), 710-761, 896.

[7] J.-P. Bourguignon, Les variétés de dimension 4 à signature non nulle dont la courbure est harmonique sont d'Einstein, Invent. Math. 63(2) (1981), 263-286.

[8] C. P. Boyer, Conformal duality and compact complex surfaces, Math. Ann. 274(3) (1986), 517-526.

[9] C. P. Boyer, A note on hyper-Hermitian four-manifolds, Proc. Amer. Math. Soc. 102(1) (1988), 157-164.

[10] N. Brotherton, Some parallelizable four-manifolds not admitting a complex structure, Bull. London Math. Soc. 10(3) (1978), 303-304.

[11] F. Campana, On twistor spaces of the class $C, J$. Differential Geom. 33(2) (1991), 541-549. 
[12] P. De Bartolomeis et A. NAnnicini, Introduction to differential geometry of twistor spaces, in : "Geometric theory of singular phenomena in partial differential equations" (Cortona, 1995), Sympos. Math. XXXVIII, Cambridge Univ. Press, Cambridge, 1998, pp. 91-160.

[13] J.-P. Demailly, Complex analytic and differential geometry, available at http ://www-fourier.ujf-grenoble.fr/ demailly/ manuscripts/agbook.pdf.

[14] S. K. Donaldson, The orientation of Yang-Mills moduli spaces and 4-manifold topology, J. Differential Geom. 26(3) (1987), $397-428$.

[15] R. Friedman Et J. W. Morgan, "Smooth four-manifolds and complex surfaces", Ergebnisse der Mathematik und ihrer Grenzgebiete (3) [Results in Mathematics and Related Areas (3)] 27, Springer-Verlag, Berlin, 1994.

[16] W. M. Goldman, Conformally flat manifolds with nilpotent holonomy and the uniformization problem for 3-manifolds, Trans. Amer. Math. Soc. 278(2) (1983), 573-583.

[17] N. J. Hitchin, Compact four-dimensional Einstein manifolds, $J$. Differential Geometry 9 (1974), 435-441.

[18] N. J. Hitchin, Kählerian twistor spaces, Proc. London Math. Soc. (3) 43(1) (1981), 133-150.

[19] M. Inoue, On surfaces of Class VII $_{0}$, Invent. Math. 24 (1974), 269-310.

[20] Y. Kamishima, Conformally flat manifolds whose development maps are not surjective. I, Trans. Amer. Math. Soc. 294(2) (1986), 607-623.

[21] M. Kato, Topology of Hopf surfaces, J. Math. Soc. Japan 27 (1975), 222-238.

[22] M. Kato, Compact differentiable 4-folds with quaternionic structures, Math. Ann. 248(1) (1980), 79-96.

[23] M. KATO, Erratum: "Compact differentiable 4-folds with quaternionic structures" [Math. Ann. 248 (1980), no. 1, 79-96; MR0569412 (81h:53037)], Math. Ann. 283(2) (1989), 352.

[24] B. KLINGLER, Structures affines et projectives sur les surfaces complexes, Ann. Inst. Fourier (Grenoble) 48(2) (1998), 441-477.

[25] K. Kodaira, On the structure of compact complex analytic surfaces. I, Amer. J. Math. 86 (1964), 751-798. 
[26] J. Lafontaine, Remarques sur les variétés conformément plates, Math. Ann. 259(3) (1982), 313-319.

[27] C. LeBrun, Topology versus Chern numbers for complex 3-folds, Pacific J. Math. 191(1) (1999), 123-131.

[28] T. Matumoto et N. Nakagawa, Explicit description of Hopf surfaces and their automorphism groups, Osaka J. Math. 37(2) (2000), 417-424.

[29] M. Pontecorvo, Uniformization of conformally flat Hermitian surfaces, Differential Geom. Appl. 2(3) (1992), 295-305.

[30] V. A. Rohlin, New results in the theory of four-dimensional manifolds, (Russian), Doklady Akad. Nauk SSSR (N.S.) 84 (1952), 221-224.

[31] S. Salamon, Special structures on four-manifolds, Conference on Differential Geometry and Topology (Italian) (Parma, 1991), Riv. Mat. Univ. Parma (4) 17* (1991), 109-123 (1993).

[32] R. Schoen, Conformal deformation of a Riemannian metric to constant scalar curvature, J. Differential Geom. 20(2) (1984), 479-495.

[33] S. Smale, Diffeomorphisms of the 2-sphere, Proc. Amer. Math. Soc. 10 (1959), 621-626.

[34] A. D. Teleman, Projectively flat surfaces and Bogomolov's theorem on class $\mathrm{VII}_{0}$ surfaces, Internat. J. Math. 5(2) (1994), 253-264.

[35] C. T. C. WALL, Geometric structures on compact complex analytic surfaces, Topology 25(2) (1986), 119-153.

[36] W.-T. Wu, "Sur les classes caractéristiques des structures fibrées sphériques", Publ. Inst. Math. Univ. Strasbourg 11, pp. 5-89, 155-156. Actualités Sci. Ind. 1183, Hermann \& Cie, Paris, 1952.

[37] S. T. YAU, Parallelizable manifolds without complex structure, Topology 15(1) (1976), 51-53.

Université de Bretagne Occidentale

6 avenue Le Gorgeu

CS 93837

29238 Brest

France

E-mail address: guillaume.deschamps@univ-brest.fr

Primera versió rebuda el 4 de juliol de 2007, darrera versió rebuda el 2 d'octubre de 2007. 